\title{
Nidification dans des cavités pierreuses de deux Megachilidae de Grèce (Epire) : Hoplitis (Hoplitis) fabrei VAN DER ZANDEN, 1987 et Megachile (Chalicodoma) manicata GIRAUD, 1861 (Hymenoptera, Megachilidae, Osmiini et Megachilini)
}

\author{
Par Gérard LE GofF *
}

Résumé. Dans cette note, je décris deux nids établis dans de petites cavités pierreuses par deux Megachilidae en Grèce : Hoplitis fabrei et Megachile manicata.

Abstract. In this note are described two nests built in little stony cavities by two Megachilidae in Greece: Hoplitis fabrei and Megachile manicata.

Mots-clés. Nids, Grèce, Megachilidae, Hoplitis fabrei, Megachile manicata.

\section{Introduction}

Au printemps 2011, un séjour en Grèce avec des amis a été essentiellement consacré à la collecte d'Apoides apiformes. Les différents sites sont situés en Epire (nord de la Grèce continentale, à proximité de la frontière avec l'Albanie), plus précisément dans la région de Konitsa.

J'ai collecté divers Megachilidae parmi lesquels Hoplitis fabrei et Megachile manicata. Mais surtout, j'ai trouvé un nid en cours de construction pour chacune de ces deux espèces.

\section{Hoplitis (Hoplitis) fabrei VAN DER ZANDEN}

Matériel collecté : Femelles : $1 \mathrm{f}$. (Kalpaki - 04.V. 2011 - N¹4319 CLG - fondatrice du nid N574), 1 f. (Igoumenitsa - 05.V.2011 - $\mathrm{N}^{\circ} 14351$ CLG), 2 f. (Kalpaki - 07.V.2011 - No14390, No14395 GLG). Mâles : 1 m. (Kalpaki - 04.V.2011 - N¹4324 CLG), 3 m. (Kalpaki - 07.V.2011 - N¹4396, N¹4464, N¹4465 CLG).

Description du nid N574 : Ce nid est construit dans une anfractuosité de grosse pierre tombée au pied d'un talus. La fondatrice a été photographiée à l'entrée de son nid dans une position de défense. La pierre brisée laisse apparaître 2 cellules complètes avec pâtée pollinique et œuf (Figure 1). La largeur du conduit a impliqué une disposition verticale des cellules $(\mathrm{H}=$

* Rue Albert Malet 44, F-76360 Barentin, France. E-mail: legoff.gerard@free.fr
$8 \mathrm{~mm} ; \mathrm{l}=5 \mathrm{~mm})$. La réserve pollinique est élaborée à partir de pollen d'Echium vulgare, d'Anchusa ondulata ou d'Anchusella cretica ces plantes étant butinées par des exemplaires capturés. Les cloisons sont faites de terre malaxée sans granules. Cette nidification est identique à celle d'Hoplitis (Hoplitis) benoisti ALFKEN. Le nid n'est pas achevé, mais l'orifice d'entrée sera sans doute obturé par un épais bouchon de terre comme le fait $H$. benoisti. Andreas Müller a intégré cette nouvelle donnée biologique sur le site consacré aux Osmiini paléarctiques (voir Müller 2012).

\section{Megachile (Chalicodoma) manicata GIRAUD}

Matériel collecté : Femelles : 2f. (Vassilopouvo 02.V.2011 - N¹4309, N¹4310 CLG), 1 f. (Stratinista 06.V.2011 - N¹4384 CLG - fondatrice du nid N577). Cette Megachile appartient au groupe Katamegachile sensu Rebmann (1970). Megachile (Chalicodoma) monstrifica Morawitz, ne diffère de $M$. manicata que par sa coloration, et serait conspécifique (et donc synonyme) de manicata (C Praz, comm. pers.).

Description du nid N577 : Ce nid, comme le précédent, est établi dans une anfractuosité d'une grosse pierre siliceuse. Il comprend deux cellules, dont une complète. Cette dernière mesure $10 / 11 \mathrm{~mm}$ de haut sur $6 \mathrm{~mm}$ de large. Elle est ovoïde et sa base est constituée par la roche elle-même (ce qui est visible sur la cellule en construction). Les parois sont relativement minces et faites de marne mêlée de granules. La provision pollinique orangée est importante et assez fluide ; elle occupe 1/3 de l'espace de la loge. La pâtée est élaborée à partir de pollen de Lotus corniculatus, sur lequel ont été capturées les 2 femelles de Vassilopoulo. 

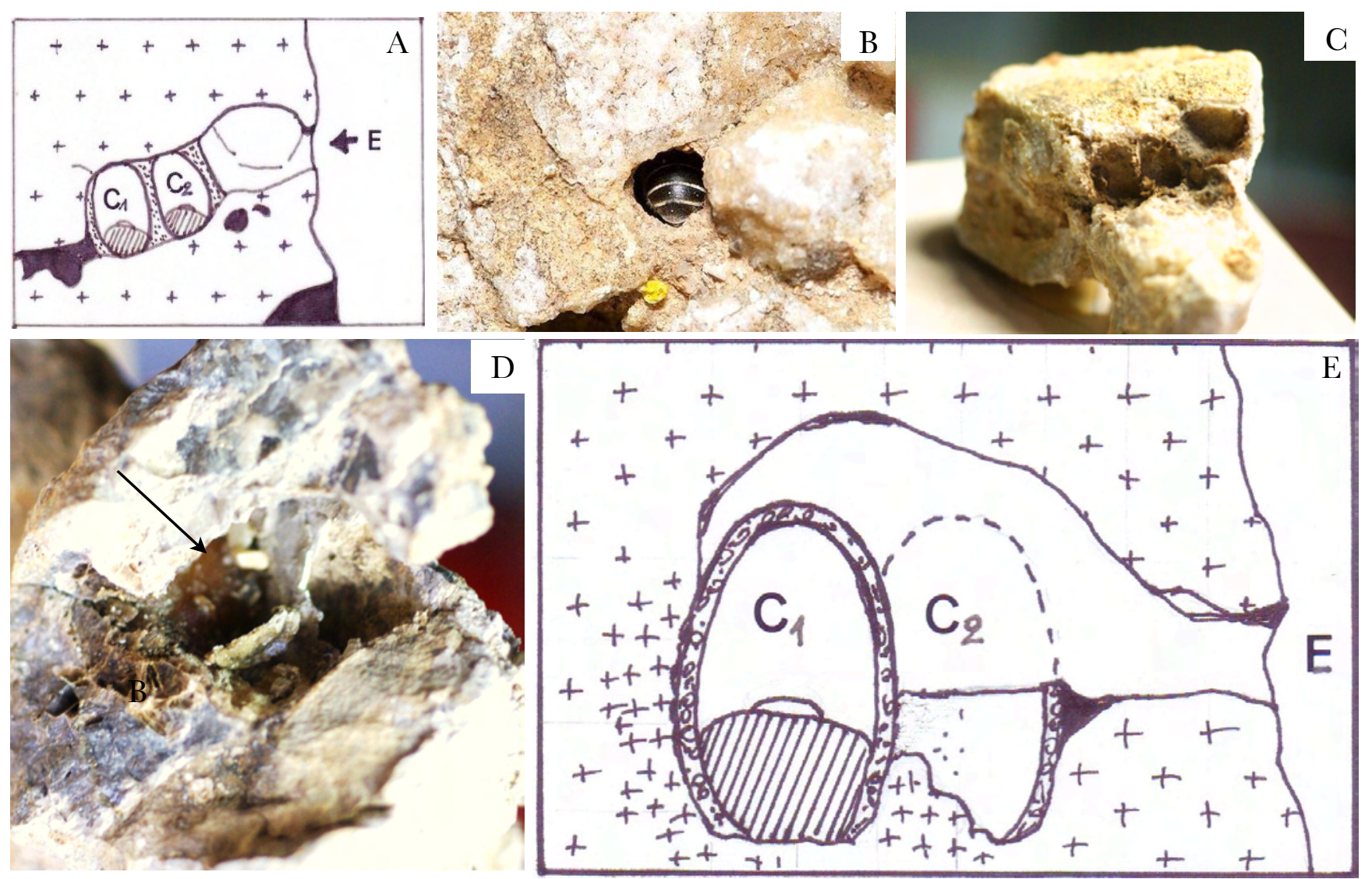

Figure 1. Etude des nids de Megachilidae découverts dans le nord de la Grèce. A. Schéma du nid N574 d'Hoplitis (Hoplitis) fabrei VAN DER ZANDEN, 1987 présentant l'entrée ("E") ainsi que les deux cellules larvaires déjà scellées (C1 et C2); B. Femelle d'H. fabrei à l'entrée de son nid (Photo NJ Vereecken); C. Détail du nid d'H. fabrei avec les deux cellules larvaires exposées, ainsi que la dernière cellule en préparation (Photo G Le Goff); D. Détail d'une cellule larvaire (C1) de Megachile (Chalicodoma) manicata GIRAUD, 1861, la flèche indique la pâtée pollinique surmontée d'un oeuf (Photo G Le Goff); E. Schéma du nid N577 de M. manicata présentant l'entrée (“E”) ainsi que les deux cellules larvaires ( $\mathrm{C} 1$ et $\mathrm{C} 2)$ dont la première était déjà scellée.

Le Lotier est aussi présent sur le site de Stratinista. L'œuf est pondu à la surface de la provision. La deuxième cellule est donc en construction et ne contient pas encore de nourriture. Le nid se limitera à ces 2 cellules. La seule inconnue reste à savoir comment le nid est clos et à l'aide de quel matériel : terre granuleuse ? pâte végétale ? inclusion de gravillons? Une chasse ponctuelle ne permet pas l'observation complète qu'on peut avoir avec des visites régulières sur des sites précis (comme j'ai pu le faire en Espagne 2004, 2005).

\section{Références bibliographiques}

Le Goff G, 2004. Note sur des Hoplitis nidifiant dans des cavités rocheuses en Espagne : Hoplitis benoisti ALFKEN et Hoplitis anthocopoides SCHENCK ; leur nidification et leurs parasites. Annexe : les Chrysidini Parasites d'Hoplitis (Hoplitis) adunca PANZER (Hymenoptera - Megachilidae : Osmiini - Leucospidae Chrysididae - Mutillidae). Lambillionea CIV 3 : 418-422.

Müller A, 2012. Palaearctic Osmiine Bees, ETH Zürich. Blog consulté le 10.vi.2012 à l'adresse http://blogs.ethz.ch/osmiini

Van der Zanden G, 1987. Neue paläarctische Taxa der Familie Megachilidae (Insecta, Hymenoptera, Apoidea). Reichenbachia 25(16): 73-83. 